\title{
Densitometric analysis of brain computed tomography as a new prognostic factor in patients with acute subdural hematoma
}

\author{
Daniel García-Pérez, MD, PhD, Irene Panero-Pérez, MD, Carla Eiriz Fernández, MD, \\ Luis Miguel Moreno-Gomez, MD, Olga Esteban-Sinovas, MD, Blanca Navarro-Main, MD, \\ Pedro A. Gómez López, MD, PhD, Ana M. Castaño-León, MD, PhD, and Alfonso Lagares, MD, PhD \\ Department of Neurosurgery, University Hospital 12 de Octubre, Madrid, Spain
}

OBJECTIVE Acute subdural hematoma (ASDH) is a major cause of mortality and morbidity after traumatic brain injury (TBI). Surgical evacuation is the mainstay of treatment in patients with altered neurological status or significant mass effect. Nevertheless, concerns regarding surgical indication still persist. Given that clinicians often make therapeutic decisions on the basis of their prognosis assessment, to accurately evaluate the prognosis is of great significance. Unfortunately, there is a lack of specific and reliable prognostic models. In addition, the interdependence of certain well-known predictive variables usually employed to guide surgical decision-making in ASDH has been proven. Because gray matter and white matter are highly susceptible to secondary insults during the early phase after TBI, the authors aimed to assess the extent of these secondary insults with a brain parenchyma densitometric quantitative $\mathrm{CT}$ analysis and to evaluate its prognostic capacity.

METHODS The authors performed a retrospective analysis among their prospectively collected cohort of patients with moderate to severe TBI. Patients with surgically evacuated, isolated, unilateral ASDH admitted between 2010 and 2017 were selected. Thirty-nine patients were included. For each patient, brain parenchyma density in Hounsfield units (HUs) was measured in 10 selected slices from the supratentorial region. In each slice, different regions of interest (ROIs), including and excluding the cortical parenchyma, were defined. The injured hemisphere, the contralateral hemisphere, and the absolute differences between them were analyzed. The outcome was evaluated using the Glasgow Outcome Scale-Extended at 1 year after TBI.

RESULTS Fifteen patients (38.5\%) had a favorable outcome. Collected demographic, clinical, and radiographic data did not show significant differences between favorable and unfavorable outcomes. In contrast, the densitometric analysis demonstrated that greater absolute differences between both hemispheres were associated with poor outcome. These differences were detected along the supratentorial region, but were greater at the high convexity level. Moreover, these $\mathrm{HU}$ differences were far more marked at the cortical parenchyma. It was also detected that these differences were more prone to ischemic and/or edematous insults than to hyperemic changes. Age was significantly correlated with the sideto-side HU differences in patients with unfavorable outcome.

CONCLUSIONS The densitometric analysis is a promising prognostic tool in patients diagnosed with ASDH. The supplementary prognostic information provided by the densitometric analysis should be evaluated in future studies.

https://thejns.org/doi/abs/10.3171/2020.4.JNS193445

KEYWORDS acute subdural hematoma; surgery; outcome; densitometric analysis; Hounsfield units; trauma; traumatic brain injury

A CUTE subdural hematoma (ASDH) is one of the most significant causes of morbidity and mortality in trauma patients. ${ }^{1,2}$ Although mortality rates are lower in more recent studies, more patients are left with poor long-term functional recovery, and the rates of good outcomes have not changed in decades., ${ }^{3,4}$
$\mathrm{CT}$ is the routine imaging modality used to define the presence and extent of injury and guide surgical planning in acute traumatic brain injury (TBI). The information supplied by the admission CT scan not only allows for diagnostic screening for potential intracranial injuries requiring acute neurosurgical interventions, but also pro-

ABBREVIATIONS ASDH = acute subdural hematoma; $A U C=$ area under the ROC curve; CRASH = Corticosteroid Randomization After Significant Head Injury; GCS = Glasgow Coma Scale; GM = gray matter; GOSE = Glasgow Outcome Scale-Extended; GWR = gray-white matter ratio; HU = Hounsfield unit; iASDH = isolated ASDH; IMPACT = International Mission for Prognosis and Analysis of Clinical Trials in TBI; IQR = interquartile range; LV = lateral ventricle; MIPAV = Medical Image Processing, Analysis, and Visualization; $\mathrm{ROC}=$ receiver operating characteristic; $\mathrm{ROI}=$ region of interest; $\mathrm{TBI}$ = traumatic brain injury; $\mathrm{WM}=$ white matter.

SUBMITTED December 19, 2019. ACCEPTED April 22, 2020.

INCLUDE WHEN CITING Published online July 31, 2020; DOI: 10.3171/2020.4.JNS193445. 
vides important prognostic information. ${ }^{5}$ There are many classification systems and prognostic models based on CT scans, such as the Marshall CT classification, ${ }^{6}$ Rotterdam CT score, ${ }^{7}$ Stockholm CT score, ${ }^{8}$ and Helsinki CT score. ${ }^{9}$ However, these prognostic models are based on the morphological features of CT scans rather than quantitative parameters.

Secondary ischemic injury could occur in patients with ASDH. Secondary cerebral ischemia is manifested as a loss of gray matter (GM) and white matter (WM) differentiation on brain $\mathrm{CT}$, which effectively predicts poor outcomes after a hypoxic-ischemic injury. ${ }^{10}$ Unfortunately, a nonnegligible variability exists because the observed decreases in the density of the GM and/or WM on CT are often very subtle. ${ }^{11}$ Minimization of inter- and intraobserver variability in the interpretation of $\mathrm{CT}$ images might be addressed by a quantitative CT analysis. As a consequence, the gray-white matter ratio (GWR) is used to quantitatively measure the degree of loss of GM-WM differentiation. ${ }^{12}$ While lower GWRs are strongly associated with worse outcomes following cardiac arrest, another report demonstrated that GWR was not associated with outcome in patients with TBI. ${ }^{13}$ A more recent and promising approach for quantitative CT analysis in patients with TBI is densitometric analysis. ${ }^{14,15}$ This approach measures changes in the overall density of the entire brain instead of relying on specific and localized regions of interest (ROIs) to investigate pathological changes in the density of brain tissues..$^{15}$

Although there are several studies assessing quantitative brain CT images in cardiac arrest or TBI, no previous study has analyzed the relationship between Hounsfield unit (HU) values and outcome in patients with ASDH. We postulated that if GM and WM densities are altered in response to secondary insults after TBI and ASDH formation, then the quantification of the density of these structures could significantly enhance the prognostic capacity of noncontrast CT images in these patients. Consequently, the aim of this study was to determine whether the densitometric analysis of the supratentorial region in patients with isolated ASDH was a reliable outcome predictor. We also aimed to compare our densitometric parameters with human operator subjective visual analysis and with existing TBI prognostication models, namely, IMPACT (International Mission for Prognosis and Analysis of Clinical Trials in TBI) ${ }^{16}$ and CRASH (Corticosteroid Randomization After Significant Head Injury). ${ }^{17}$

\section{Methods}

\section{Study Design and Timeline}

We performed an 8-year (2010-2017) retrospective analysis of our prospectively maintained database of all patients with moderate to severe TBI admitted to our level I trauma center (Supplemental Fig. 1).

\section{Inclusion Criteria}

Because other traumatic intracranial lesions are usually concomitant to ASDH and might influence the unfavorable outcome of this disease,,$^{18,19}$ we aimed to identify selective predictive factors of poor prognosis among pa- tients with surgically treated isolated ASDH (iASDH). We defined iASDH as the main lesion when the volume was $\geq$ $10 \mathrm{~cm}^{3}$ and represented $\geq 90 \%$ of intracranial hematoma on the CT scan that revealed the worst pattern and severity of structural brain damage after head injury. Thus, we included all adult patients ( $\geq 15$ years old) with traumatic iASDH (which was evacuated) as the main lesion on the CT scan. All patients suffered from blunt severe or moderate TBI, as defined by a score of $<13$ points on the Glasgow Coma Scale (GCS) following a nonsurgical resuscitation or after deterioration to this score in the first 48 hours after TBI.

\section{Exclusion Criteria}

Patients were excluded if they experienced a penetrating-missile TBI, if CT acquisition was delayed for more than 48 hours after TBI, or they recovered to a GCS score $\geq 13$ after cessation of sedation or the effects of CNS depressors. Bilateral or posterior fossa ASDHs were excluded. Subarachnoid hemorrhage, epidural hematomas, intraparenchymal hematomas, or hemorrhagic brain contusions representing more than $10 \%$ of hemorrhagic lesions were also excluded. The different bleeding volumes were calculated using volumetric CT-based software (see Supplemental Materials and Methods).

\section{Nonoperated Patient Cohort}

We included a further cohort from the same time interval that had an ASDH that met the inclusion criteria but did not undergo surgical treatment. Clinical, radiological, and surgical features of all patients are found in the Supplemental Materials and Methods.

\section{Quantitative Densitometric Analysis}

All CT images were evaluated by an observer (D.G.P.) who was not aware of the clinical situation or outcome of the patients. We acquired CT scans on a Philips Brilliance 16 CT scanner (Philips Healthcare) with 3-mm slices. We used only noncontrast CT scans of the head for the study. The tube voltage was $120 \mathrm{kVp}$ and the tube current was $167 \mathrm{~mA}$. All images were acquired in the axial mode (image matrix size $512 \times 512$ ). Patients were excluded from the analyses if $\mathrm{CT}$ images were missing or the parameters could not be calculated due to a significant artifact on the images.

Image analysis was performed with the Medical Image Processing, Analysis, and Visualization (MIPAV) application (version 7.4.0, NIH; http://mipav.cit.nih.gov/). Predefined head CT lookup tables in MIPAV were employed. Brain parenchyma density was measured in 10 selected slices from the supratentorial region: we manually selected the first slice (just above the tentorium) and the last one (at the high convexity level), and the other slices were equally distributed along the axial axis (Fig. 1A-J). ROIs were defined semiautomatically using the level-set ROI tool. In this method, MIPAV software first analyzes the intensity values and uses the results from its level-set algorithm to determine the probable boundary of the ROI, and then generates a contour. Two ROI contours were developed on each slice: first, an ROI included the brain paren- 

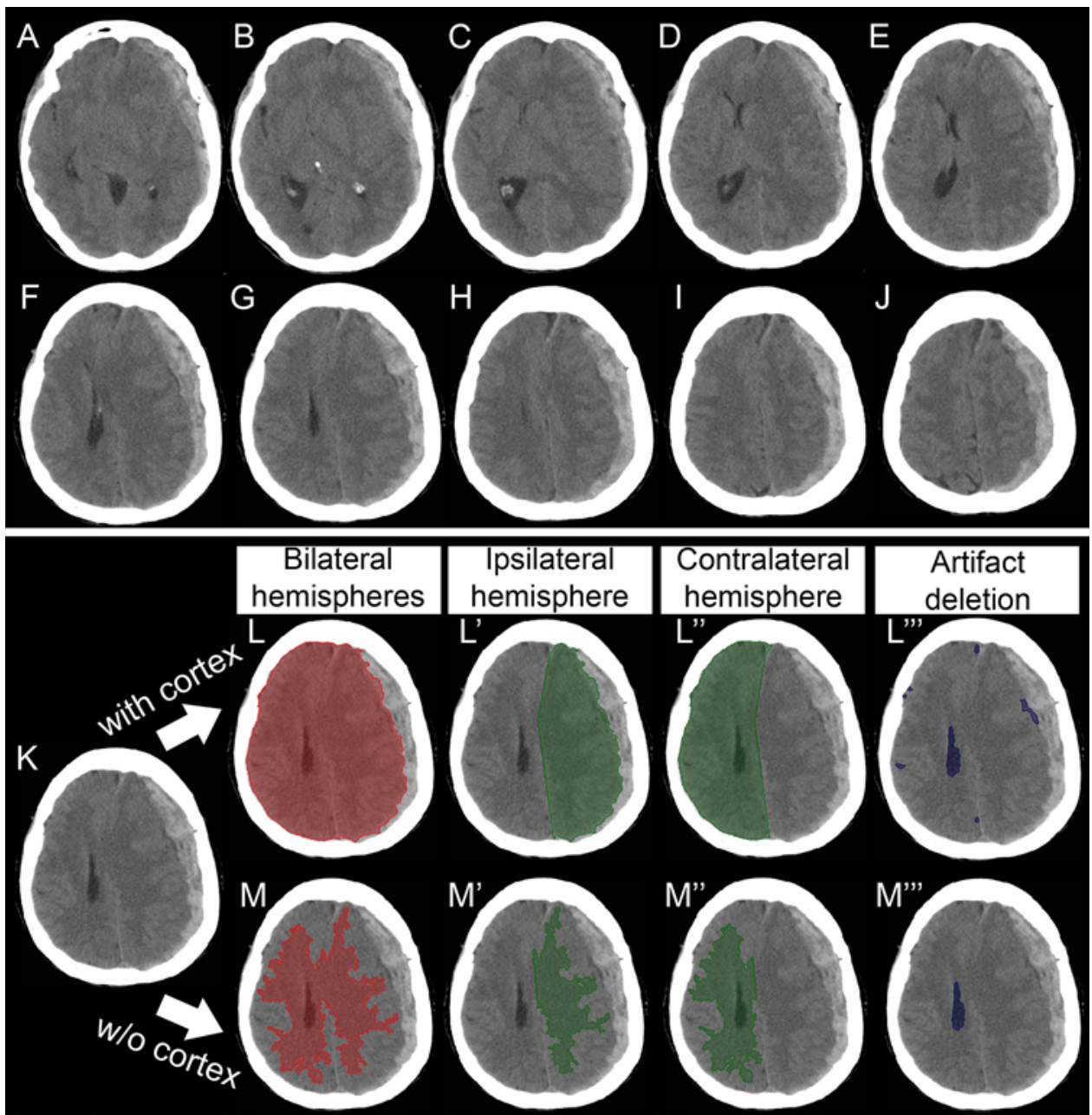

FIG. 1. Brain parenchyma density was measured in 10 selected axial slices from the supratentorial region. A-J: Head CT slices from a selected patient. A large left frontoparietal ASDH is observed. We manually selected the first slice (just above the tentorium, A) and the last one (at the high convexity level, J), and the other slices were equally distributed along the axial axis. K-M: A CT image (G) was selected to demonstrate our densitometric analysis. An ROI that included the whole brain parenchyma and excluded the ASDH was defined semiautomatically $(\mathrm{L})$. The ROI was then divided into the two hemispheres, taking into account the midline shift. L' represents the hemisphere ipsilateral to the ASDH, and L" exemplifies the contralateral hemisphere. As an additional step, the observer excluded the pixels that were not appropriate for the analysis (L'"). These included, but were not restricted to, CSF of the ventricles or sulci, calcifications, hemorrhagic lesions, or other artifacts. These pixels were selected under a semiautomatic method and then subtracted for the analysis from the previous ROls. At the same slice, a second ROI that excluded the cortical gray matter was developed (M). The same method as explained above was performed (M'-M"'). Figure is available in color online only.

chyma and excluded the ASDH (Fig. 1L), and the second ROI also excluded the cortical gray matter (Fig. 1M). As depicted in Fig. 1, each ROI was then divided into the two hemispheres, taking into account the midline shift. As an additional step, the observer excluded the pixels from the analysis that were not appropriate for the analysis; these included, but were not restricted to, CSF of the ventricles or sulci, calcifications, hemorrhagic lesions, or other artifacts. These pixels were selected under a semiautomatic method and then subtracted from the previous ROIs.

The median \pm interquartile range (IQR) was calculated for each ROI. The densitometric analysis included the hemisphere ipsilateral to the hematoma and the contralateral hemisphere. In addition, we also calculated the absolute difference between both hemispheres for each patient (contralateral - ipsilateral). A global densitometric measure (which included the 10 slices) was calculated. A subgroup analysis that included slices 1-4 (basal ganglia level), 5-7 (lateral ventricle [LV] level), and 8-10 (high convexity level) was also performed (Fig. 2).

\section{Human Operator Visual Densitometric Analysis}

All CT images were then evaluated by the senior author (A.L.), who was not aware of the clinical outcome of the patients or the quantitative densitometric analysis results. The observer visualized the entire brain CT scan and decided if both hemispheres were isodense or not. Moreover, if there were differences in the brain parenchyma density, the observer decided which hemisphere was more hypodense. 


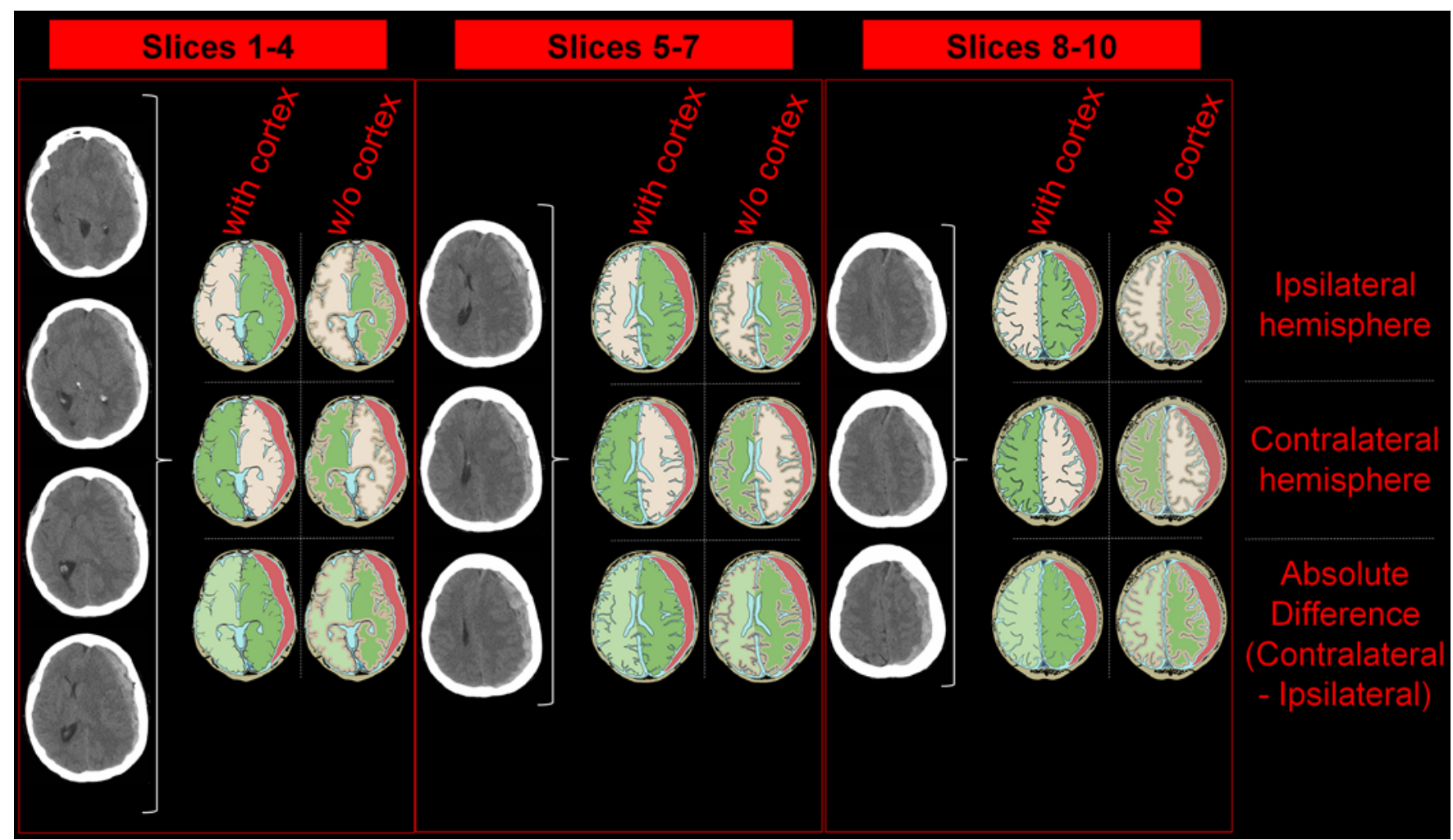

FIG. 2. Head CT slices showing an example of the brain parenchyma measurements in HUs. According to the method illustrated in Fig. 1, a global densitometric measure (which included the 10 slices) was calculated. In addition, a subgroup analysis that included slices 1-4 (basal ganglia level), 5-7 (LV level), and 8-10 (high convexity level) was also performed. For each region, the whole parenchyma (including the cortex) densitometric analysis included the hemisphere ipsilateral to the hematoma, the contralateral hemisphere, and the absolute difference between both hemispheres (contralateral - ipsilateral). Then, the same analysis was performed with the ROls that excluded the cortical parenchyma. Figure is available in color online only.

\section{Outcome Assessment}

The functional outcome for surgically treated patients was evaluated with a structured interview 12 months after the TBI by the Glasgow Outcome Scale-Extended (GOSE). ${ }^{20}$ GOSE scores of 5-8 were deemed favorable and 1-4 as unfavorable. The cohort of patients who did not undergo surgical treatment was divided into 3 groups: early death ( $\leq 72$ hours after hospital admission), late death at hospital ( $>72$ hours), and patients who were discharged.

\section{Statistical Analysis}

Due to the relatively small size of the cohorts, nonparametric analyses were performed. Quantitative variables are presented as the median $\pm \mathrm{IQR}$ and qualitative variables are expressed as absolute frequency and its relative percentage. For 2-group comparisons, the Mann-Whitney U-test and chi-square or Fisher exact tests were used as appropriate. To evaluate the operated and nonoperated groups, the Kruskal-Wallis test was used to compare the 4 groups. The Dunn-Bonferroni post hoc method was further employed for any dependent variables for which the Kruskal-Wallis test was significant.

A $p$ value $<0.05$ was considered statistically significant. For the densitometric data analysis, we performed 12 comparisons (4 different numbers of slices, ipsilateral favorable vs unfavorable outcome, contralateral favorable vs unfavorable, and hemisphere difference favorable vs unfavorable). Thus, we controlled the familywise error rate with the Bonferroni correction. The $\mathrm{p}$ level for significance was lowered to $0.05 / 12=0.0042$.

The prognostic capacities of brain density parameters were further evaluated by analyzing a receiver operating characteristic (ROC) curve. The Spearman's correlation coefficient was used to measure the association between the densitometric values and age. Two of the most frequently used prognostication models for TBI, IMPACT ${ }^{16}$ and $\mathrm{CRASH},{ }^{17}$ were compared to the prognostic performance of the densitometric parameters to evaluate the efficacy of the proposed parameters. Kappa coefficients were calculated for assessing the reliability between the quantitative densitometric analysis and the human operator visual densitometric analysis. All statistical analyses were conducted using SPSS (version 22.0, IBM Corp.).

\section{Results}

Forty-one patients met the inclusion/exclusion criteria. Two of the patients were excluded because their images contained a significant artifact. Thus, 39 patients were deemed eligible for further analyses and were ultimately included in the study. Twenty-eight patients $(71.8 \%)$ were male, and the median patient age was 43 years (IQR 28 
TABLE 1. Clinical and radiographic characteristics of surgically treated ASDH patients experiencing either good or poor functional outcomes at 12 months

\begin{tabular}{|c|c|c|c|}
\hline Characteristic & Favorable & Unfavorable & p Value \\
\hline No. of patients & 15 & 24 & \\
\hline Age, yrs & & & 0.8922 \\
\hline Median & 41.0 & 44.0 & \\
\hline IQR & $26.0-75.0$ & $31.75-58.75$ & \\
\hline Males (\%) & $10(66.7)$ & $18(75)$ & 0.718 \\
\hline GCS admission score (full range) & & & 0.270 \\
\hline Median & 4 & 3 & \\
\hline IQR & $3-9$ & $3-5$ & \\
\hline GCS admission score $\leq 8(\%)$ & $11(73.3)$ & $22(91.7)$ & 0.180 \\
\hline Admission motor score $\leq 4$ (\%) & $10(66.7)$ & $20(83.3)$ & 0.266 \\
\hline Pupillary abnormalities (\%) & $7(46.7)$ & $15(62.5)$ & 0.508 \\
\hline Hematoma volume, $\mathrm{cm}^{3}$ & & & 0.6739 \\
\hline Median & 62.24 & 55.76 & \\
\hline IQR & $45.92-102.9$ & $38.21-99.23$ & \\
\hline Midline shift, mm & & & 0.1924 \\
\hline Median & 10.0 & 12.0 & \\
\hline IQR & $6.0-14.0$ & $6.5-15$ & \\
\hline Hypoxia (\%) & $0(0)$ & $3(12.5)$ & 0.271 \\
\hline Hypotension (\%) & $2(13.3)$ & $7(29.2)$ & 0.437 \\
\hline Major extracranial injury (\%) & $4(26.7)$ & $8(33.3)$ & 0.734 \\
\hline
\end{tabular}

60 years). Thirty-three (84.6\%) of 39 patients presented with GCS scores $\leq 8$. Fifteen patients $(38.5 \%)$ had a favorable outcome.

Traditional prognostic factors described in the TBI literature were evaluated. ${ }^{21-23}$ We analyzed if these characteristics (age, GCS score, motor score, pupillary abnormality, midline shift, and/or hematoma volume) differed between groups. As shown in Table 1, none of the parameters showed a meaningful difference between the favorable and unfavorable groups.

The tissue density on the CT images was measured in HUs. As shown in Table 2, no differences in the ipsilateral hemisphere were observed for the densitometric analysis with cortex, or for the whole supratentorial region (slices 1-10), or for the different specific subregions (slices 1-4 [basal ganglia level], 5-7 [LV level], or 8-10 [high convexity level]). Similarly, when we studied the contralateral hemisphere, no differences were detected. In contrast, greater HU differences between both hemispheres were strongly correlated with worse outcomes (Table 2; Fig. $3 \mathrm{~A})$.

As depicted in Table 3, the densitometric analysis excluding the cortex revealed that no differences in the ipsilateral or contralateral hemispheres were observed between outcome groups. The worse outcome group exhibited a significantly greater $\mathrm{HU}$ difference between both hemispheres when the whole supratentorial region was analyzed (Fig. 3B). However, no differences were detected when the different subregions were selected.

The power of the densitometric parameters in predict-
TABLE 2. Assessment of CT parameters in the whole brain parenchyma in the favorable and unfavorable outcome groups

\begin{tabular}{|c|c|c|c|}
\hline \multirow[b]{2}{*}{ With Cortex } & \multicolumn{2}{|c|}{ Hematoma Side } & \multirow{2}{*}{$\begin{array}{c}\text { Absolute Difference } \\
\text { (contralateral - } \\
\text { ipsilateral) }\end{array}$} \\
\hline & Ipsilateral & Contralateral & \\
\hline \multicolumn{4}{|l|}{ All slices } \\
\hline \multicolumn{4}{|l|}{ Favorable } \\
\hline Median & 37.47 & 37.14 & 0.2648 \\
\hline IQR & $36.20-39.60$ & $36.14-39.37$ & $0.1125-0.5412$ \\
\hline \multicolumn{4}{|c|}{ Unfavorable } \\
\hline Median & 37.57 & 37.66 & 0.6594 \\
\hline IQR & $35.76-38.46$ & $35.79-39.08$ & $0.3843-1.2162$ \\
\hline$p$ value & 0.638 & 0.989 & 0.001 \\
\hline \multicolumn{4}{|l|}{ Slices 1-4 } \\
\hline \multicolumn{4}{|l|}{ Favorable } \\
\hline Median & 38.29 & 37.95 & 0.2761 \\
\hline IQR & $36.07-40.08$ & $36.02-40.46$ & $0.1918-0.6966$ \\
\hline \multicolumn{4}{|l|}{ Unfavorable } \\
\hline Median & 38.15 & 38.17 & 0.6855 \\
\hline IQR & $36.64-39.06$ & $36.35-39.27$ & $0.3074-1.1931$ \\
\hline$p$ value & 0.765 & 0.989 & 0.051 \\
\hline \multicolumn{4}{|l|}{ Slices 5-7 } \\
\hline \multicolumn{4}{|l|}{ Favorable } \\
\hline Median & 37.08 & 36.82 & 0.4776 \\
\hline IQR & $35.91-39.14$ & $35.97-38.82$ & $0.1706-0.7391$ \\
\hline \multicolumn{4}{|c|}{ Unfavorable } \\
\hline Median & 37.02 & 37.21 & 0.8577 \\
\hline IQR & $34.39-37.75$ & $35.35-38.61$ & $0.5951-1.6960$ \\
\hline$p$ value & 0.466 & 0.921 & 0.011 \\
\hline \multicolumn{4}{|l|}{ Slices 8-10 } \\
\hline \multicolumn{4}{|l|}{ Favorable } \\
\hline Median & 37.44 & 36.94 & 0.3055 \\
\hline IQR & $35.23-39.15$ & $35.41-39.46$ & $0.1047-0.5083$ \\
\hline \multicolumn{4}{|c|}{ Unfavorable } \\
\hline Median & 37.91 & 37.71 & 0.8589 \\
\hline IQR & $35.67-38.61$ & $35.60-39.24$ & $0.4850-1.1489$ \\
\hline$p$ value & 0.966 & 0.875 & 0.000 \\
\hline
\end{tabular}

All data given in HUs, except for $p$ values. The Mann-Whitney U-test was used to compare groups. A p value $<0.0042$ was considered statistically significant. Boldface type indicates statistical significance.

ing the prognostic outcome was further assessed via ROC analyses (Fig. 4). The whole brain (slices 1-10) with cortex densitometric analysis exhibited the highest prognostic capacity (Fig. 4B). The densitometric analysis with cortex at the high convexity region (slices 8-10) also exhibited a high predictive power for worse outcomes (Fig. 4C). When the cortex was excluded from the ROIs, the whole region (slices 1-10) analysis still effectively distinguished patients with unfavorable outcomes (Fig. 4D and E). In contrast, densitometric parameters of the different subregions without cortex did not effectively distinguish the outcome groups. The area under the ROC curve (AUC) values for the densitometric parameters are summarized 

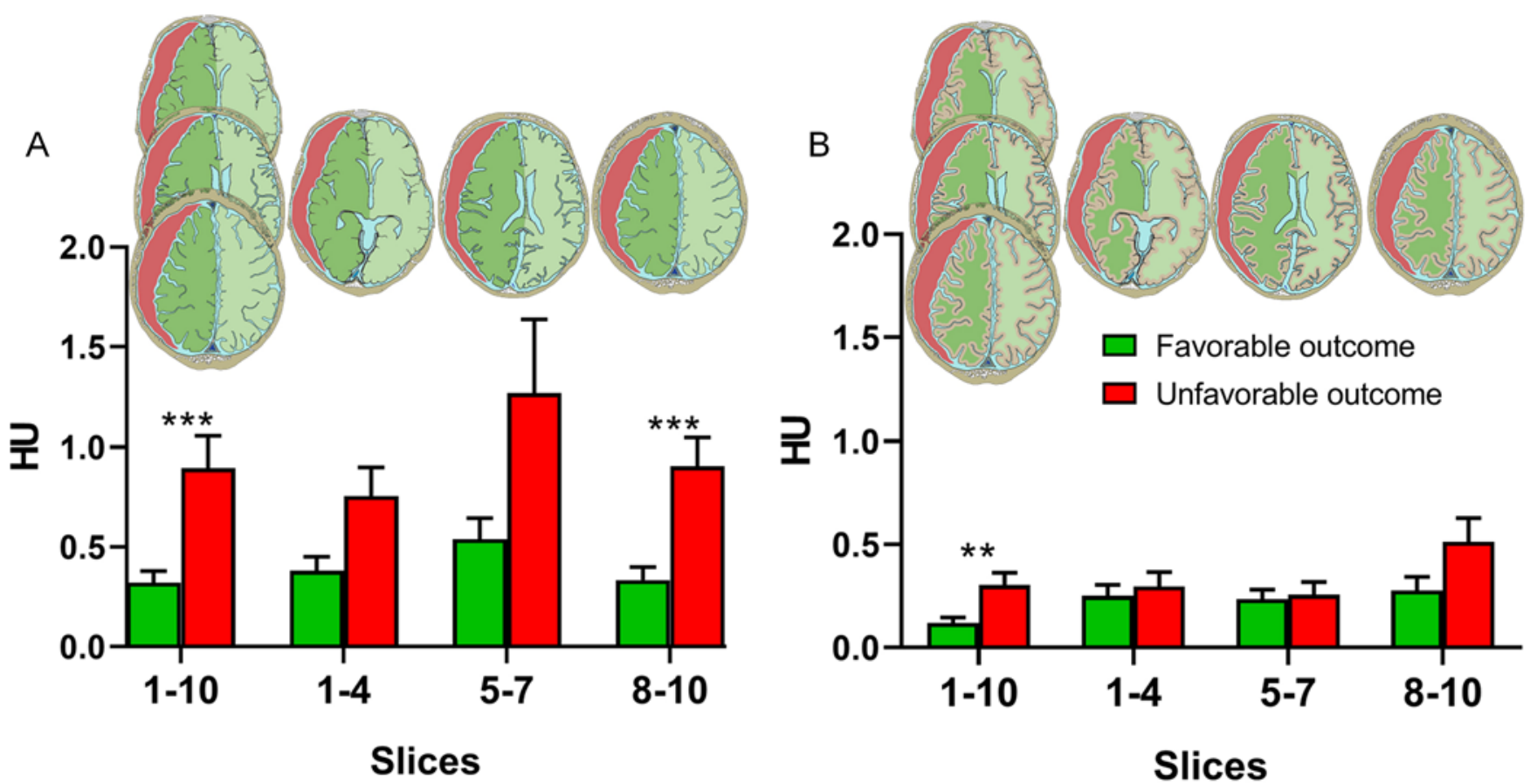

FIG. 3. HU differences between both hemispheres were strongly associated with worse outcomes. A: The densitometric analysis including the cortex revealed that significant differences were detected for the whole supratentorial region (slices 1-10), but also for the different specific subregions (slices 1-4 [basal ganglia level], 5-7 [LV level], or 8-10 [high convexity level]). B: The densitometric analysis excluding the cortex showed that the worse outcome group exhibited a significantly greater HU difference between both hemispheres only when the whole supratentorial region was analyzed. However, no differences were detected when the different subregions were selected. ${ }^{* *} p<0.01,{ }^{* *} p<0.001$. Figure is available in color online only.

in Supplementary Table 1. Regarding the densitometric parameters showing the highest statistically significant differences, the optimal cutoff points on the ROC curves were calculated, and the performances of the cutoff points in predicting the outcomes were further evaluated. A difference between both hemispheres $>0.571$ HUs (slices 1-10) and $>0.629$ HUs (slices 8-10) achieved a $70.8 \%$ sensitivity and $86.7 \%$ specificity, respectively.

Once we had established that greater HU differences between both hemispheres were strongly correlated with worse outcomes and that these differences were more pronounced at cortical areas, we analyzed the direction of these differences. Lower density tissue exhibits a lower HU value and vice versa. As shown in Fig. 5, patients with unfavorable outcome showed greater differences between both hemispheres. The majority of patients exhibited reduced HUs in the ipsilateral hemisphere to the hematoma side (Fig. 5A and B). The decrease in HUs might indicate the presence of a severe ischemic and/or edematous insult. However, a small proportion of patients with unfavorable outcome showed increased HU values in the ipsilateral hematoma, suggesting that hyperemic changes might also occur. These differences were markedly reduced when the cortex was excluded from the densitometric analysis (Fig. $5 \mathrm{C}$ and D).

The age-induced difference in the density distribution was also investigated, which could have influenced our findings. We did not find a relationship between the age of the patients and the mean HU values obtained for the ip- silateral or contralateral hemispheres (data not shown). In contrast, we detected that age was significantly correlated with the side-to-side $\mathrm{HU}$ differences between hemispheres in patients with unfavorable outcome (Fig. 5E), thus suggesting that older patients are more vulnerable to these secondary insults. As expected, this relationship was no longer observed in patients with favorable outcome (Fig. $5 \mathrm{~F})$, where secondary insults were reduced or absent.

\section{Predictive Power of Existing TBI Prognostic Models}

The predictive capacity of the existing TBI prognostic models (i.e., IMPACT and CRASH models) was compared to that of the densitometric parameters (Supplemental Table 1). The IMPACT model showed an adequate performance when compared with the CRASH model. However, some densitometric parameters, particularly those measuring the brain parenchyma with cortex (slices 1-10 and 8-10), outperformed the IMPACT and CRASH models.

\section{Visual Densitometric Analysis}

For the blinded observer, 15 patients exhibited isodense hemispheres. Conversely, the observer found differences between hemispheres in 24 patients. Most of the time (in 22 of 24 patients), the hemisphere ipsilateral to the hematoma appeared to be more hypodense.

For the purpose of statistical analysis, the interhemispheric quantitative densitometric differences were dichotomized. The cutoff value was chosen so that half of the 
TABLE 3. Assessment of CT parameters without cortex in the favorable and unfavorable outcome groups

\begin{tabular}{|c|c|c|c|}
\hline \multirow[b]{2}{*}{ Without Cortex } & \multicolumn{2}{|c|}{ Hematoma Side } & \multirow{2}{*}{$\begin{array}{c}\text { Absolute Difference } \\
\text { (contralateral - } \\
\text { ipsilateral) }\end{array}$} \\
\hline & Ipsilateral & Contralateral & \\
\hline \multicolumn{4}{|l|}{ All slices } \\
\hline \multicolumn{4}{|l|}{ Favorable } \\
\hline Median & 33.37 & 33.41 & 0.1096 \\
\hline IQR & $32.53-35.20$ & $32.51-34.93$ & $0.0265-0.2023$ \\
\hline \multicolumn{4}{|l|}{ Unfavorable } \\
\hline Median & 33.66 & 33.54 & 0.2639 \\
\hline IQR & $32.39-34.42$ & $32.22-34.52$ & $0.1274-0.3286$ \\
\hline$p$ value & 0.989 & 0.943 & 0.003 \\
\hline \multicolumn{4}{|l|}{ Slices 1-4 } \\
\hline \multicolumn{4}{|l|}{ Favorable } \\
\hline Median & 34.19 & 34.42 & 0.1666 \\
\hline IQR & $32.88-36.41$ & $32.86-36.21$ & $0.1371-0.3045$ \\
\hline \multicolumn{4}{|l|}{ Unfavorable } \\
\hline Median & 34.63 & 34.46 & 0.1847 \\
\hline IQR & $33.81-35.70$ & $33.51-35.52$ & $0.0899-0.4257$ \\
\hline$p$ value & 0.853 & 0.921 & 0.966 \\
\hline \multicolumn{4}{|l|}{ Slices 5-7 } \\
\hline \multicolumn{4}{|l|}{ Favorable } \\
\hline Median & 33.05 & 33.02 & 0.1854 \\
\hline IQR & $32.16-34.84$ & $32.22-34.65$ & $0.0912-0.3223$ \\
\hline \multicolumn{4}{|l|}{ Unfavorable } \\
\hline Median & 32.68 & 32.83 & 0.1793 \\
\hline IQR & $31.32-34.00$ & $31.30-34.28$ & $0.0690-0.4781$ \\
\hline$p$ value & 0.466 & 0.484 & 0.921 \\
\hline \multicolumn{4}{|l|}{ Slices 8-10 } \\
\hline \multicolumn{4}{|l|}{ Favorable } \\
\hline Median & 32.55 & 32.57 & 0.2324 \\
\hline IQR & 31.34-34.19 & $31.35-34.33$ & $0.1142-0.3343$ \\
\hline \multicolumn{4}{|l|}{ Unfavorable } \\
\hline Median & 32.88 & 33.22 & 0.3643 \\
\hline IQR & $31.09-34.45$ & $30.93-34.01$ & $0.1846-0.8025$ \\
\hline$p$ value & 0.875 & 0.966 & 0.097 \\
\hline
\end{tabular}

All data given in HUs, except for $p$ values. The Mann-Whitney U-test was used to compare groups. A $p$ value $<0.0042$ was considered statistically significant. Boldface type indicates statistical significance.

patients were included in the "no interhemispheric differences" group and the other half in the "interhemispheric differences" group. Human operator visual densitometric analysis showed poor agreement with the quantitative densitometric analysis, given that kappa indices were always below 0.20 (Supplemental Table 2). Therefore, humanoperated visual estimation of side-to-side differences in density was not valid or reliable.

\section{Quantitative Densitometric Analysis in Nonoperated Patients}

The tissue density on the CT images was further ana- lyzed in a cohort of nonoperated patients. For the statistical analysis, these patients were divided into two groups: the early death group, and another group that included the patients who died in the hospital after 72 hours of admission (due to the ASDH or medical complications) and patients who were discharged from the hospital. As shown in Supplemental Table 3, no differences in the ipsilateral or contralateral hemispheres were observed between the different groups. Interestingly, we detected that greater $\mathrm{HU}$ differences between both hemispheres were observed both in operated patients with unfavorable outcome and in early death nonoperated patients, when compared to favorable outcome after surgical evacuation. Similar results were observed when the densitometric analysis excluded the cortex, although these did not reach statistical significance ( $\mathrm{p}=0.007$; Supplemental Table 4).

\section{Discussion}

The main objective of this study was to evaluate the prognostic capacity of changes in the brain CT density after an ASDH occurs. Despite modern medical care, morbidity and mortality in traumatic ASDH surgery remains dramatically high and ranges between $40 \%$ and $70 \% .^{18,24,25}$ Importantly, decreased neurological status (expressed as GCS score) at clinical presentation has largely been found to be associated with impaired clinical evolution in ASDH patients. ${ }^{26-30}$ The outcome of adult patients suffering from ASDH evacuation in our series is similar to recent neurosurgical series concerning this issue..$^{24,28}$ However, as shown in Table 1, none of the classic demographic, clinical, or radiographic prognostic parameters ${ }^{21-23}$ showed a meaningful difference between the favorable and unfavorable groups. Furthermore, the interdependence of certain well-known predictive variables has been suggested. ${ }^{31}$ Therefore, rather than being independent features, their importance may be related to their status as surrogate measures of a more fundamental underlying clinical feature, such as the intracranial mass effect severity.

TBI is a complex disease that causes structural damage and functional deficits that are due to both primary and secondary injury mechanisms. ${ }^{32}$ The clinical paradox in which the outcome for a given clinical state appears to be much worse with ASDH than with extradural hematomas suggests that an additional insult may be responsible for poor prognosis in patients with ASDH. For these reasons, methods designed to assess the extent of the secondary ischemic-edematous insults during the acute phase of ASDH and enhance the diagnostic and prognostic capacities of CT are needed.

\section{Densitometric Analysis for Predicting Favorable Outcome in Patients With ASDH}

Quantitative CT analysis, such as GWR, is well established as a reliable prognostic method following cardiac

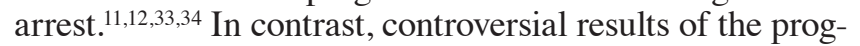
nostic capacity of the GWR in TBI have been reported to date. ${ }^{13,15,27}$ The decrease in the densities of both the deep GM and WM was evident in the groups with worse outcomes after TBI, which could explain the ineffectiveness of the GWR. ${ }^{15}$ 

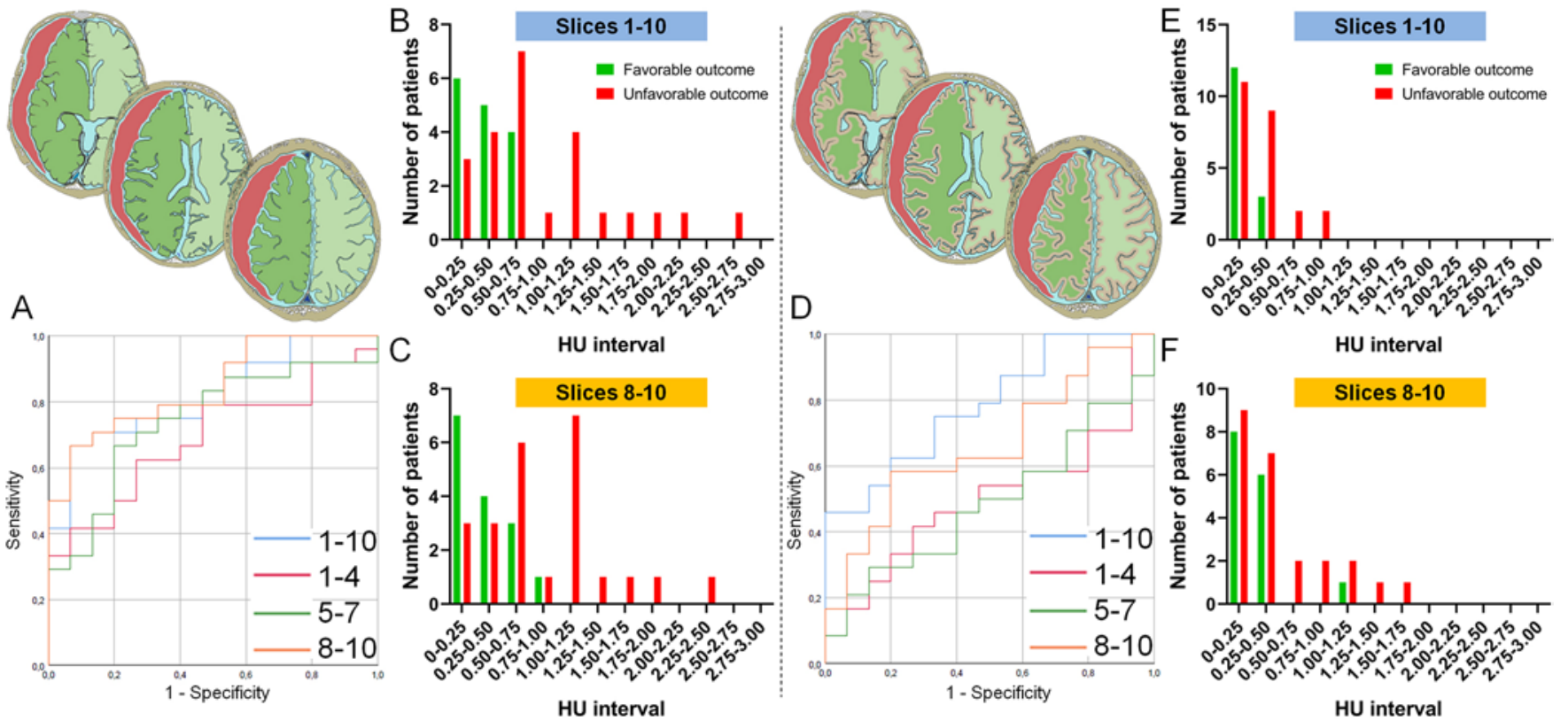

FIG. 4. The power of the densitometric parameters in predicting the prognostic outcome was further assessed via ROC analyses. A: A nonparametric ROC curve for the densitometric analysis including the cortex. B and C: Bar charts showing the distribution of the absolute differences between both hemispheres in the densitometric analysis including the cortex (B, slices 1-10; $C$, slices 8-10). D: A nonparametric ROC curve for the densitometric analysis excluding the cortex. E and F: Bar charts showing the distribution of the absolute differences between both hemispheres in the densitometric analysis excluding the cortex ( $E$, slices 1-10; F, slices 8-10). The AUC values for the densitometric parameters are summarized in Supplemental Table 1. Figure is available in color online only.

A different approach for quantitative CT analysis of patients with TBI is the densitometric analysis..$^{14,15}$ One of the most important advantages of densitometric analysis is that instead of relying on specific localized ROIs to investigate pathological changes in the density of brain tissues, this approach measures changes in the overall density of the entire brain. Other authors have developed a completely automated protocol that performs a quantitative examination of the parenchymal density distribution, thereby enabling subsequent measurements of the proportions of GM and WM showing a normal preestablished density. ${ }^{14,15}$

In the present study, rather than analyzing predesignated pixels of interest or developing a densitometry analysis based on predefined values, we compared the mean density of the entire supratentorial brain for patients with ASDH. Our densitometric protocol has some similarities and differences with the work published by Kim and colleagues. ${ }^{14,15}$ First, our protocol is a nonautomated one, which might be tedious and time-consuming. To address this limitation we did not measure $\mathrm{HU}$ values of the whole brain. Instead, we selected 10 slices from the supratentorial region: we manually selected the first slice (just above the tentorium) and the last one (at the high convexity level), and the other slices were equally selected along the axial axis. With our methodology, the average time spent to segment all ROIs in an individual case was approximately 60 minutes for each case. Of outmost importance, besides the overall density of the entire supratentorial brain, our protocol allowed us to differentiate which region of the brain (basal ganglia, bodies of the LV, or high convexity level) played a more important role in our observations.
Our standardized protocol also allowed us to measure the exact contribution of the cortical gray matter to the differences we observed. The use of different $\mathrm{CT}$ machines and image acquisition parameters affects the $\mathrm{HU}$ values of the acquired CT images. Therefore, another important advantage of our protocol is that each patient is his or her own control (by comparing the ipsilateral hemisphere against the contralateral hemisphere). Additionally, brain density is significantly affected by the age of the patient. Thus, having this intrapatient control allowed us to avoid bias related to age-related changes in HU values. Our study demonstrates that $\mathrm{HU}$ values measured at the initial CT are a reliable outcome predictor in patients with ASDH. To the best of our knowledge, this is the first study to assess whole brain parenchyma CT density in patients with ASDH.

Our model showed an AUC larger than many published predictive models in the TBI population. ${ }^{15,35,36}$ We believe that the high discrimination power of our model was attributable to the high volume of data collected from head CT images, and the narrow focus on a subpopulation of patients with iASDH. Our results also support the idea that geriatric patients are more susceptible to the secondary insult after an ASDH.

\section{Pathophysiological Implications of Altered Brain Parenchyma Density}

Secondary mechanisms are responsible for much of the brain damage that occurs in ASDH. The reasons for the specific biological reactions involving the brain beneath 


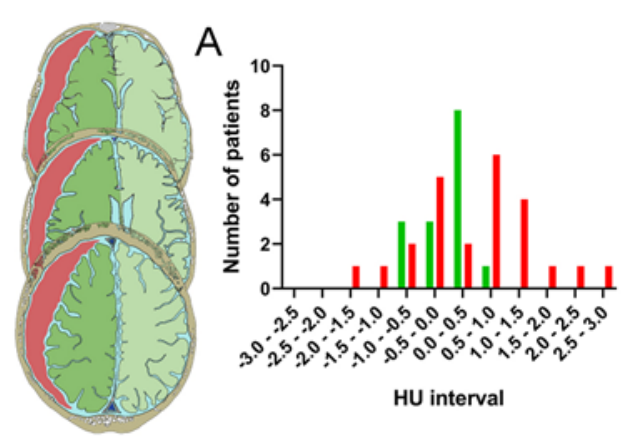

$B$
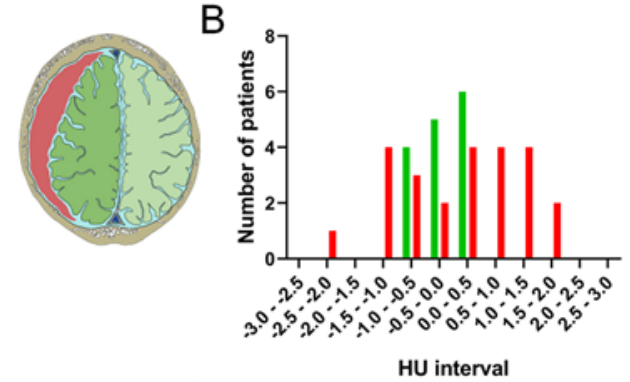
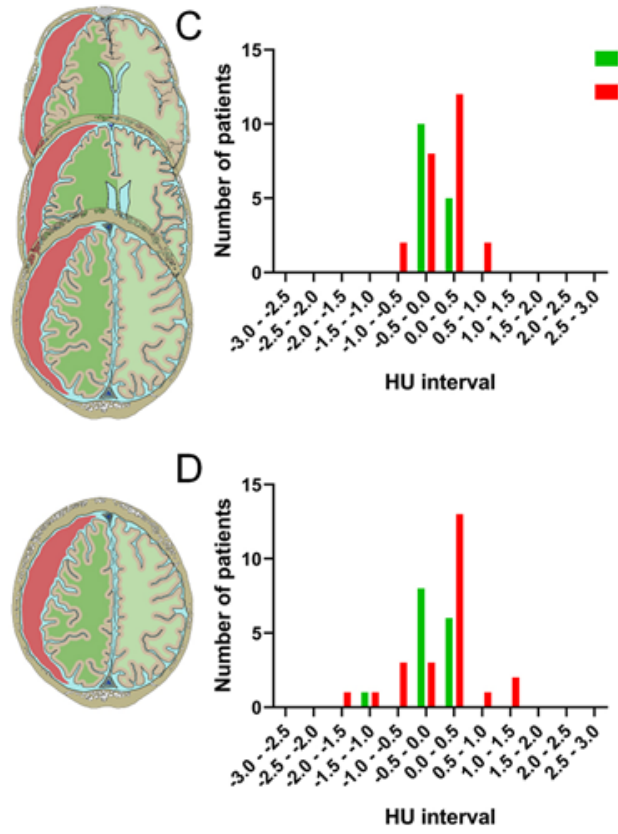

Hyperemia Ischemia/edema

Favorable outcome

Unfavorable outcome

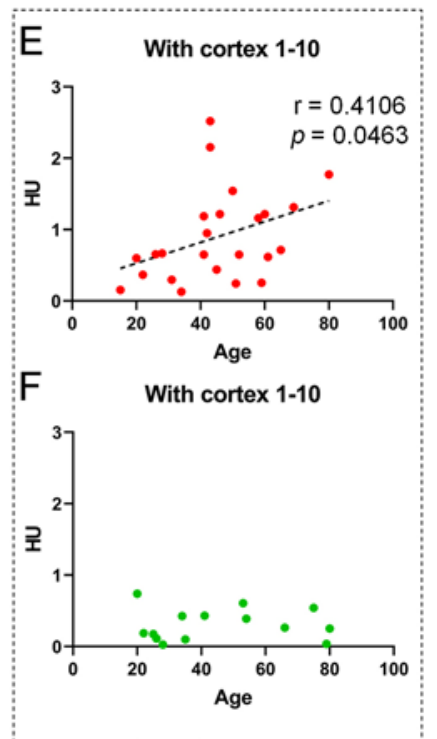

FIG. 5. A and B: Bar charts showing the trend of the HU differences between both hemispheres in the densitometric analysis including the cortex (A, slices 1-10; B, slices 8-10). The majority of patients exhibited reduced HUs in the ipsilateral hemisphere to the hematoma side compared to the contralateral side, thus indicating the presence of a severe ischemic and/or edematous insult. However, a small proportion of patients with unfavorable outcome showed increased HU values in the ipsilateral hematoma, suggesting that hyperemic changes might also occur. $\mathbf{C}$ and $\mathbf{D}$ : These differences were markedly reduced when the cortex was excluded from the densitometric analysis (C, slices 1-10; D, slices 8-10). E and F: The Spearman's correlation coefficient was used to measure the association between the densitometric values and age. Age was significantly correlated with the side-to-side $\mathrm{HU}$ differences between hemispheres in patients with unfavorable outcome $(\mathrm{E})$. Figure is available in color online only.

the ASDH may be manifold and can be discussed here only speculatively. It is well known that CT attenuation and brain water content are inversely correlated. ${ }^{37}$ One of the most frequent effects of cerebral ischemia is cerebral edema, which is a major cause of brain swelling. According to our results, it is more likely that the local effects of the subdural hematoma initiate a sequence of events leading to focal ischemia, with consequent brain edema and swelling, which were detected as a decrease in HU values on initial CT in the damaged hemisphere. It also appears that early pathophysiological pathways are activated to a larger extent at the high convexity level, thus causing more pronounced $\mathrm{CT}$ changes at this brain level. The magnitude of side-to-side differences entails the severity of insult from cerebral edema and is closely related to worse outcome. However, such a subtle decrease is nearly impossible to perceive with the human eye, as confirmed in this work.

Some of the patients with unfavorable outcome exhibited higher CT brain density in the ipsilateral injury hemisphere compared to the noninjured hemisphere. Increased CT density after head injury might be related to an increased cerebral blood volume. ${ }^{38-40}$ The development of global cerebral hyperemia in TBI is often associated with elevated ICP and an unfavorable outcome. ${ }^{41}$ Importantly, a previous report found that hyperemic values predomi- nated in the cortex located in the damaged hemisphere of patients with an evacuated ASDH and were related to an unfavorable outcome. ${ }^{42}$ Thus, our data support the contention that a small proportion of patients displayed brain hyperemia, which was also associated with a worse outcome. In addition, hyperemia was more frequent at the high convexity level and only seen at the cortical GM in our study.

\section{Limitations}

Certain limitations of the study should be mentioned. First, this is a retrospective, single-center study with a small sample size. Therefore, the findings presented here may not be generalizable to other institutions with varying patient populations or clinical practices. Furthermore, because this was an observational study, we were not able to control for selection bias in terms of which patients were offered surgical intervention. Only a small proportion of patients were administered neuropsychological testing during their rehabilitation period. Unfortunately, the sample size is too small to draw conclusions. Future studies should incorporate neuropsychological testing, which might yield more sensitive and precise assessment of functional level and function recovery over time. A further shortcoming of the current study is that the determination of HU values of the whole brain using our method is tedious and time-consuming and not practical for surgical 
decision-making. However, we believe that this analysis provides valuable information for clinicians to accurately assess the prognosis of the patients and thus contribute to better inform the families. In addition, we detected that the densitometric analysis at the high convexity level (slices 8-10) had an equivalent high accuracy in discriminating between patients who had a favorable outcome and those who did not when compared to the whole brain analysis (slices 1-10), which speeds the CT analysis. Our study should be regarded as hypothesis-generating work that warrants further investigation and needs validation with an external data set before using this method in clinical practice.

\section{Conclusions}

This study demonstrated the utility of quantitative CT image analysis in the context of ASDH. The magnitude of side-to-side CT density differences between the lesioned and nonlesioned hemispheres was revealed as the key factor. A difference $>0.571$ HUs for the whole supratentorial region and $>0.629$ HUs for the high convexity region was highly correlated with poor outcome. Although the densitometric analysis remains to be validated, this method shows its potential to be used in routine practice during the early stages of TBI.

\section{Acknowledgments}

This research was jointly funded by the ISCIII and FEDER European institutions, with FIS project no. PI18/01387. The sponsor had no role in the design or conduct of this research.

\section{References}

1. Bullock MR, Chesnut R, Ghajar J, et al. Surgical management of acute subdural hematomas. Neurosurgery. 2006; 58(3)(suppl):S16-S24, Si-Siv.

2. Karibe H, Hayashi T, Hirano T, et al. Surgical management of traumatic acute subdural hematoma in adults: a review. Neurol Med Chir (Tokyo). 2014;54(11):887-894.

3. Frontera JA, Egorova N, Moskowitz AJ. National trend in prevalence, cost, and discharge disposition after subdural hematoma from 1998-2007. Crit Care Med. 2011;39(7): 1619-1625.

4. Leitgeb J, Mauritz W, Brazinova A, et al. Outcome after severe brain trauma due to acute subdural hematoma. J Neurosurg. 2012;117(2):324-333.

5. Thelin EP, Nelson DW, Vehviläinen J, et al. Evaluation of novel computerized tomography scoring systems in human traumatic brain injury: an observational, multicenter study. PLoS Med. 2017;14(8):e1002368.

6. Marshall LF, Marshall S, Klauber MR, et al. A new classification of head injury based on computerized tomography. $J$ Neurosurg. 1991;75(suppl):S14-S20.

7. Maas AI, Hukkelhoven CW, Marshall LF, Steyerberg EW. Prediction of outcome in traumatic brain injury with computed tomographic characteristics: a comparison between the computed tomographic classification and combinations of computed tomographic predictors. Neurosurgery. 2005;57(6): 1173-1182.

8. Nelson DW, Nyström H, MacCallum RM, et al. Extended analysis of early computed tomography scans of traumatic brain injured patients and relations to outcome. J Neurotrauma. 2010;27(1):51-64.

9. Raj R, Siironen J, Skrifvars MB, et al. Predicting outcome in traumatic brain injury: development of a novel computerized tomography classification system (Helsinki computerized tomography score). Neurosurgery. 2014;75(6):632-647.

10. Heinz UE, Rollnik JD. Outcome and prognosis of hypoxic brain damage patients undergoing neurological early rehabilitation. BMC Res Notes. 2015;8:243.

11. Choi SP, Park HK, Park KN, et al. The density ratio of grey to white matter on computed tomography as an early predictor of vegetative state or death after cardiac arrest. Emerg Med J. 2008;25(10):666-669.

12. Torbey MT, Selim M, Knorr J, et al. Quantitative analysis of the loss of distinction between gray and white matter in comatose patients after cardiac arrest. Stroke. 2000;31(9): 2163-2167.

13. Jin XQ, Huang YW, Yang MF. Association between graywhite matter ratio in computed tomography and outcome in patients with extra-axial hematoma. World Neurosurg. 2018; 116:e759-e765.

14. Kim H, Kim GD, Yoon BC, et al. Quantitative analysis of computed tomography images and early detection of cerebral edema for pediatric traumatic brain injury patients: retrospective study. BMC Med. 2014;12:186.

15. Kim H, Kim YT, Song ES, et al. Changes in the gray and white matter of patients with ischemic-edematous insults after traumatic brain injury. J Neurosurg. 2019;131(4):12431253.

16. Steyerberg EW, Mushkudiani N, Perel P, et al. Predicting outcome after traumatic brain injury: development and international validation of prognostic scores based on admission characteristics. PLoS Med. 2008;5(8):e165.

17. Perel P, Arango M, Clayton T, et al. Predicting outcome after traumatic brain injury: practical prognostic models based on large cohort of international patients. BMJ. 2008;336(7641): $425-429$.

18. Baucher G, Troude L, Pauly V, et al. Predictive factors of poor prognosis after surgical management of traumatic acute subdural hematomas: a single-center series. World Neurosurg. 2019;126:e944-e952.

19. Koç RK, Akdemir H, Oktem IS, et al. Acute subdural hematoma: outcome and outcome prediction. Neurosurg Rev. 1997;20(4):239-244.

20. Wilson JT, Pettigrew LE, Teasdale GM. Structured interviews for the Glasgow Outcome Scale and the extended Glasgow Outcome Scale: guidelines for their use. J Neurotrauma. 1998;15(8):573-585.

21. Chesnut RM, Marshall LF, Klauber MR, et al. The role of secondary brain injury in determining outcome from severe head injury. J Trauma. 1993;34(2):216-222.

22. Eisenberg HM, Gary HE Jr, Aldrich EF, et al. Initial CT findings in 753 patients with severe head injury. A report from the NIH Traumatic Coma Data Bank. J Neurosurg. 1990; 73(5):688-698.

23. Signorini DF, Andrews PJ, Jones PA, et al. Predicting survival using simple clinical variables: a case study in traumatic brain injury. J Neurol Neurosurg Psychiatry. 1999;66(1): 20-25.

24. Ryan CG, Thompson RE, Temkin NR, et al. Acute traumatic subdural hematoma: current mortality and functional outcomes in adult patients at a Level I trauma center. J Trauma Acute Care Surg. 2012;73(5):1348-1354.

25. Servadei F, Nasi MT, Giuliani G, et al. CT prognostic factors in acute subdural haematomas: the value of the 'worst' CT scan. Br J Neurosurg. 2000;14(2):110-116.

26. Hamed M, Schuss P, Daher FH, et al. Acute traumatic subdural hematoma: surgical management in the presence of cerebral herniation - a single-center series and multivariate analysis. World Neurosurg. 2016;94:501-506.

27. Jin XQ, Du XF, Yang MF, Zhang Q. Development and validation of prognostic model for patients with acute subdural 
hematoma-reliable nomogram. World Neurosurg. 2018;124: e266-e275.

28. Kim KH. Predictors for functional recovery and mortality of surgically treated traumatic acute subdural hematomas in 256 patients. J Korean Neurosurg Soc. 2009;45(3):143-150.

29. Kotwica Z, Brzeziński J. Acute subdural haematoma in adults: an analysis of outcome in comatose patients. Acta Neurochir (Wien). 1993;121(3-4):95-99.

30. Petridis AK, Dörner L, Doukas A, et al. Acute subdural hematoma in the elderly; clinical and CT factors influencing the surgical treatment decision. Cent Eur Neurosurg. 2009;70(2): 73-78.

31. Yuh EL, Cooper SR, Ferguson AR, Manley GT. Quantitative CT improves outcome prediction in acute traumatic brain injury. J Neurotrauma. 2012;29(5):735-746.

32. Wajima D, Sato F, Kawamura K, et al. Venous or arterial blood components trigger more brain swelling, tissue death after acute subdural hematoma compared to elderly atrophic brain with subdural effusion (SDE) model rats. Brain Res. 2017;1670:165-172.

33. Gentsch A, Storm C, Leithner C, et al. Outcome prediction in patients after cardiac arrest: a simplified method for determination of gray-white matter ratio in cranial computed tomography. Clin Neuroradiol. 2015;25(1):49-54.

34. Metter RB, Rittenberger JC, Guyette FX, Callaway CW. Association between a quantitative CT scan measure of brain edema and outcome after cardiac arrest. Resuscitation. 2011; 82(9):1180-1185.

35. Hukkelhoven CW, Rampen AJ, Maas AI, et al. Some prognostic models for traumatic brain injury were not valid. $J$ Clin Epidemiol. 2006;59(2):132-143.

36. Hukkelhoven CW, Steyerberg EW, Habbema JD, et al. Predicting outcome after traumatic brain injury: development and validation of a prognostic score based on admission characteristics. J Neurotrauma. 2005;22(10):1025-1039.

37. Dzialowski I, Weber J, Doerfler A, et al. Brain tissue water uptake after middle cerebral artery occlusion assessed with CT. J Neuroimaging. 2004;14(1):42-48.

38. Bruce DA, Alavi A, Bilaniuk L, et al. Diffuse cerebral swelling following head injuries in children: the syndrome of "malignant brain edema”. J Neurosurg. 1981;54(2):170-178.

39. Enevoldsen EM, Cold G, Jensen FT, Malmros R. Dynamic changes in regional $\mathrm{CBF}$, intraventricular pressure, $\mathrm{CSF} \mathrm{pH}$ and lactate levels during the acute phase of head injury. $J$ Neurosurg. 1976;44(2):191-214.
40. Obrist WD, Langfitt TW, Jaggi JL, et al. Cerebral blood flow and metabolism in comatose patients with acute head injury. Relationship to intracranial hypertension. J Neurosurg. 1984; 61(2):241-253.

41. Kelly DF, Kordestani RK, Martin NA, et al. Hyperemia following traumatic brain injury: relationship to intracranial hypertension and outcome. J Neurosurg. 1996;85(5):762-771.

42. Chieregato A, Noto A, Tanfani A, et al. Hyperemia beneath evacuated acute subdural hematoma is frequent and prolonged in patients with an unfavorable outcome: a Xe-computed tomographic study. Neurosurgery. 2009;64(4):705-718.

\section{Disclosures}

The authors report no conflict of interest concerning the materials or methods used in this study or the findings specified in this paper.

\section{Author Contributions}

Conception and design: García-Pérez, Lagares. Acquisition of data: García-Pérez, Gómez López, Castaño-León, Lagares. Analysis and interpretation of data: García-Pérez. Drafting the article: García-Pérez. Critically revising the article: all authors. Reviewed submitted version of manuscript: all authors. Approved the final version of the manuscript on behalf of all authors: García-Pérez. Statistical analysis: García-Pérez, Castaño-León, Lagares. Study supervision: Gómez López, Lagares.

\section{Supplemental Information \\ Online-Only Content}

Supplemental material is available with the online version of the article.

Supplemental Materials. https://thejns.org/doi/suppl/10.3171/ 2020.4.JNS193445.

\section{Correspondence}

Daniel García-Pérez: University Hospital 12 de Octubre, Madrid, Spain.dgp.neurosurgery@gmail.com. 